\title{
Correction to: On Bernstein's inequality for polynomials
}

\author{
H. Queffélec ${ }^{1} \cdot$ R. Zarouf $\mathbf{Z}^{2,3}$
}

Received: 17 April 2019 / Accepted: 17 April 2019 / Published online: 8 May 2019

(c) Springer Nature Switzerland AG 2019

\section{Correction to: Analysis and Mathematical Physics https://doi.org/10.1007/s13324-019-00294-x}

In this addendum to the paper On Bernstein's inequality for polynomials [Anal. Math. Phys. online 20 March 2019], we rectify the beginning of Section 5 where we mentioned a proof of Mahler's result, i.e. the case $p=0$ in Bernstein's inequality, using subharmonicity. In particular, we take into account a reference that we previously missed, and that Paul Nevai, whom we thank, has very recently brought to our attention.

\section{Case $p=0$, Mahler's result}

This section (as well as Section 6) owes much to old conversations with Nazarov [3] during the fall of 1994, when the first-named author was finishing the joint work [4] with B. Saffari. F. Nazarov then emphasized the importance of subharmonicity. The possible use of this subharmonicity was first alluded to by the referee (M. Marden) of Mahler's 1961 paper [2]. But then it was also intensively used in the 1989 paper [1], which fully reproved Bernstein's inequality in $L^{0}$ and then in $L^{p}, 0<p \leq \infty$. The authors of the implied survey paper were not aware of [1] when their work was accepted in "Analysis and Mathematical Physics" and were informed of this important

The original article can be found online at https://doi.org/10.1007/s13324-019-00294-x.

$凶 \quad$ R. Zarouf

rachid.zarouf@univ-amu.fr

H. Queffélec

Herve.Queffelec@univ-lille.fr

1 USTL, Laboratoire Paul Painlevé U.M.R. CNRS 8524 et Fédération CNRS Nord-Pas-de-Calais FR 2956, Université Lille Nord de France, 59655 Villeneuve d'Ascq Cedex, France

2 Laboratoire Apprentissage, Didactique, Evaluation, Formation, Aix-Marseille Université, 32 Rue Eugène Cas CS 90279, 13248 Marseille Cedex 04, France

3 Department of Mathematics and Mechanics, Saint Petersburg State University, 28, Universitetski pr., St. Petersburg, Russia 198504 
paper by P. Nevai, whom they thank, very shortly after their work appeared in arXiv. They apologize for this overview.

\section{References}

1. von Golitschek, A., Lorentz, G.G.: Bernstein's inequalities in $L_{p}, 0 \leq p \leq \infty$, Rocky Mountain. J. Math. 19, 145-156 (1989)

2. Mahler, K.: On the zeros of the derivative of a polynomial. Proc. R. Soc. Lond. Ser. A 264, 145-154 (1961)

3. Nazarov, F.: Private communication (1994)

4. Queffélec, H., Saffari, B.: On Bernstein's inequality and Kahane's ultraflat polynomials. J. Fourier Anal. Appl. 2(6), 519-582 (1996)

Publisher's Note Springer Nature remains neutral with regard to jurisdictional claims in published maps and institutional affiliations. 